\title{
VECTOR BUNDLES TRIVIALIZED BY PROPER MORPHISMS AND THE FUNDAMENTAL GROUP SCHEME
}

\author{
INDRANIL BISWAS AND JOÃO PEDRO P. DOS SANTOS
}

\begin{abstract}
Let $X$ be a smooth projective variety defined over an algebraically closed field $k$. Nori constructed a category of vector bundles on $X$, called essentially finite vector bundles, which is reminiscent of the category of representations of the fundamental group (in characteristic zero). In fact, this category is equivalent to the category of representations of a pro-finite group scheme which controls all finite torsors. We show that essentially finite vector bundles coincide with those which become trivial after being pulled back by some proper and surjective morphism to $X$.
\end{abstract}

\section{INTRODUCTION}

Let $k$ be an algebraically closed field, and let $X / k$ be a smooth projective variety. By a vector bundle we mean a locally free coherent sheaf. We are interested in studying vector bundles on $X$ enjoying the following property:

(T) There exists a proper $k$-scheme $Y$ together with a surjective (proper) morphism $f: Y \longrightarrow X$ such that the pull-back $f^{*} E$ is trivial.

Note that we can, and usually will, assume that $Y$ is a proper variety: just replace $Y$ by the reduced subscheme underlying an irreducible component of $Y$ dominating $X$.

In [9], Nori introduced the category of essentially finite vector bundles on $X$; this category "is" the category of representations of a pro-finite group scheme which generalizes (respectively equals) the étale fundamental group of SGA1 in positive characteristic (respectively characteristic zero). A vector bundle $E \longrightarrow X$ is essentially finite if and only if there exists a finite group scheme $G$, a $G$-torsor $f: P \longrightarrow X$, and a representation $V$ of $G$, such that $E=P \times{ }^{G} V$. (This is not Nori's original definition, but one of the main consequences of [9].) Since the $G$-torsor $f^{*} P$ is canonically trivialized, the pull-back $f^{*} E$ is trivializable, so that essentially finite vector bundles enjoy property $(\mathrm{T})$. We establish a converse, which should also be regarded as a generalization of a result due to Lange and Stuhler [5, Proposition 1.2] stating that a vector bundle trivialized by a finite étale

2000 Mathematics Subject Classification. 14L15, 14F05.

Key words and phrases. Essentially finite vector bundle, fundamental group scheme, trivialization. 
covering comes from a representation of the étale fundamental group. The reader can also regard what follows in connection with Proposition 1.2 of [SGA 1, X]; of course, the point here is not to impose conditions on the trivializing morphism other than properness and surjectivity.

Theorem 1. Let $X$ be a smooth and projective variety over the algebraically closed field $k$. A vector bundle $E$ over $X$ is essentially finite if and only if it satisfies property (T).

Property (T) of vector bundles is in fact stronger than it appears to be. Here is what we mean by this. Let $f: Y \longrightarrow X$ be a proper and surjective morphism and $E \longrightarrow X$ a vector bundle such that $f^{*} E$ is trivial. Consider the Stein factorization of $f$

$$
Y \stackrel{f^{\prime}}{\longrightarrow} Z=\operatorname{Spec}\left(f_{*} \mathcal{O}_{Y}\right) \stackrel{g}{\longrightarrow} X
$$

Using the fact that $f_{*}^{\prime} \mathcal{O}_{Y}=\mathcal{O}_{Z}$, it follows that if $V \longrightarrow Z$ is a vector bundle such that $\left(f^{\prime}\right)^{*} V$ is trivial, then $V$ itself is trivial. Hence, $g^{*} E$ is trivial. This shows that a vector bundle $E$ over $X$ satisfies property (T) if and only if it satisfies the following condition:

(TF) There exists a finite and surjective morphism $g: Z \longrightarrow X$ such that $g^{*} E$ is trivial.

This allows us to state an equivalent version of Theorem 1;

Theorem 2. Let $X$ be a smooth and projective variety over the algebraically closed field $k$. A vector bundle $E$ over $X$ is essentially finite if and only if it satisfies property (TF).

Remarks: (a) Let $f: Y \longrightarrow X$ be a surjective morphism of finite type such that $f^{*} E \cong \mathcal{O}_{Y}^{\oplus r}$. For each $x: \operatorname{Spec}(k) \longrightarrow X$, we pick $y: \operatorname{Spec}(k) \longrightarrow Y$ satisfying $f \circ y=x$. We have $x^{*} E=y^{*} f^{*} E$, hence $x \mapsto \operatorname{dim}_{k} x^{*} E=r$ is a constant function on the closed points of $X$. As $X$ is of finite type, it must be a constant function [4, p. 125, Ex. 5.8(a)]. We then apply [4, p. 125, Ex. 5.8(c)] to conclude that $E$ is a vector bundle.

(b) Below, see the remarks on page 9, we observe that Theorem 1 is true in characteristic zero even if we only require $X$ to be normal.

(c) If $k$ is the algebraic closure of a finite field, then Theorem 1 is a direct consequence of Proposition 4 and Maruyama's conjecture proved by Langer [6].

1.1. Some notations and conventions. As before, $k$ stands for an algebraically closed field. A variety is an integral and separated scheme of finite type over $k$. Here $X$ will always stand for a projective smooth variety over $k$. The dimension of $X$ is denoted by $d$. For any scheme $Y$, the category of vector bundles over $Y$ will be denoted by $\mathbf{V B}(Y)$. 
By $L$ we will denote a very ample line bundle on $X$. Degrees of vector bundles are always taken with respect to the polarization $L$. The rational number

$$
\mu(E):=\frac{c_{1}(L)^{d-1} \cap c_{1}(E)}{\operatorname{rank}(E)}=\frac{\operatorname{degree}(E)}{\operatorname{rank}(E)}
$$

is called the slope of $E$.

Tannakian categories. We will follow the conventions of [3]. All Tannakian categories will be over the field $k$, and if $\omega: \mathcal{T} \longrightarrow k-\bmod$ is a fiber functor, we will denote by $\pi(\mathcal{T}, \omega)$ the corresponding group scheme [3, Theorem 2.11], [9].

\section{TANNAKIAN PROPERTIES}

We use the formalism of Tannakian categories to study property (T).

\subsection{The category of objects with property (T).}

Definition 3. (i) Let $\mathcal{T}(X)$ denote the full subcategory of $\mathbf{V B}(X)$ whose objects are vector bundles satisfying property $(\mathrm{T})$, or equivalently, property $(\mathrm{TF})$.

(ii) Let $f: Y \longrightarrow X$ be a surjective and proper morphism. We will denote by $\mathcal{T}_{Y}(X)$ the full subcategory of $\mathcal{T}(X)$ whose objects become trivial when pulled back to $Y$.

A vector bundle $E \longrightarrow X$ is called Nori-semistable if, for every pair $(C, \alpha)$, where $C / k$ is a smooth projective curve and $\alpha: C \longrightarrow X$ is a morphism, the pull-back $\alpha^{*} E$ is semistable of degree zero.

Proposition 4. Let $E \in \mathcal{T}(X)$. Then $E$ is Nori-semistable.

Proof. Let $\alpha: C \longrightarrow X$ be a morphism from a curve. Let $f: Y \longrightarrow X$ be a surjective proper morphism such that $f^{*} E$ is trivial. We can find a smooth and projective curve $C^{\prime} / k$ and morphisms $\beta: C^{\prime} \longrightarrow Y$ and $\nu: C^{\prime} \longrightarrow C$ such that $f \circ \beta=\alpha \circ \nu$ and $\nu$ is surjective. Hence $\nu^{*} \alpha^{*} E$ is trivial. This implies that $\alpha^{*} E$ is semistable of degree zero.

Corollary 5. (i) Let $f: Y \longrightarrow X$ be a proper surjective morphism from a variety $Y$. Then $\mathcal{T}_{Y}(X)$ is abelian.

(ii) The category $\mathcal{T}(X)$ is abelian.

(iii) Let $Y$ be as before, $E, Q$ be vector bundles of degree zero, and $\alpha: E \longrightarrow Q$ be an epimorphism. Then, if $f^{*} E$ is trivial, so is $f^{*} Q$. In particular, the subcategory $\mathcal{T}_{Y}(X)$ is stable under quotients in $\mathcal{T}(X)$. 
Proof. (i) Let $\alpha: E \longrightarrow F$ be an arrow of $\mathcal{T}_{Y}(X)$. From the assumptions, we conclude that $\operatorname{Ker}\left(f^{*}(\alpha)\right), \operatorname{Im}\left(\varphi^{*}(\alpha)\right)$ and $\operatorname{Coker}\left(f^{*}(\alpha)\right)$ are all trivial. Since $E$ and $F$ are Norisemistable, it follows that $\operatorname{Ker}(\alpha) \operatorname{Im}(\alpha)$ and $\operatorname{Coker}(\alpha)$ are all vector bundles [9, Lemma 3.6]. It is then straightforward to see that $f^{*}$ commutes with $\operatorname{Ker}(\alpha)$ and $\operatorname{Coker}(\alpha)$ so that the kernel and cokernel of $\alpha$ are in $\mathcal{T}_{Y}(X)$.

Part (ii) can be easily deduced from (i), since every pair $E, E^{\prime} \in \mathcal{T}(X)$ belong to $\mathcal{T}_{Y}(X)$ for some $Y$ which is integral.

To prove part (iii), let $q$ and $n$ be the ranks of $Q$ and $E$ respectively. Let $\operatorname{Gr}(q, n)$ be the Grassmannian parametrizing linear subspaces of codimension $q$ in $\mathbb{A}^{n}$. We let $\mathcal{U}$ be the universal quotient of $\mathcal{O}_{\operatorname{Gr}(q, n)}^{\oplus n}$, so that the line bundle $\operatorname{det}(\mathcal{U}) \longrightarrow \operatorname{Gr}(q, n)$ is very ample [8]. The hypothesis on $E$ allows us to define a morphism

$$
\gamma: Y \longrightarrow \operatorname{Gr}(q, n)
$$

such that $\gamma^{*} \mathcal{U}=f^{*} Q$. Unless the dimension of the image of $\gamma$ is zero, the ampleness of $\operatorname{det}(\mathcal{U})$ contradicts the assumption $\operatorname{deg}(Q)=0$. Hence $\gamma$ is a trivial morphism, and thereby $f^{*} Q$ is trivial.

It is clear that if $E, F$ are objects of $\mathcal{T}(X)$ (respectively, $\mathcal{T}_{Y}(X)$ ), then $E \otimes_{\mathcal{O}_{X}} F$ is also an object of $\mathcal{T}(X)$ (respectively, $\mathcal{T}_{Y}(X)$ ). This endows $\mathcal{T}(X)$ (respectively, $\mathcal{T}_{Y}(X)$ ) with a $k$-linear monoidal structure.

Corollary 6. The category $\mathcal{T}(X)$ is Tannakian over $k$. If $x_{0}$ is a $k$-rational point, then taking the fiber at $x_{0}$ defines an exact and faithful tensor functor $x_{0}^{*}: \mathcal{T}(X) \longrightarrow k-$ mod.

2.2. Reformulation of Theorem 1 in terms of the category $\mathcal{T}(X)$. In order to state the next result, we need to introduce some terminology and recall some well known results from the theory of Tannakian categories.

Definition 7. Let $(\mathcal{T}, \otimes)$ be a Tannakian category over $k$ and $V$ an object of $\mathcal{T}$. The monodromy category of $V$, or the Tannakian subcategory generated by $V$, is the full subcategory

$$
\langle V ; \mathcal{T}\rangle_{\otimes}
$$

of $\mathcal{T}$ admitting as objects the sub-quotients of all generalized tensor powers

$$
\left[V^{\otimes a_{1}} \otimes\left(V^{\vee}\right)^{\otimes b_{1}}\right] \oplus \cdots \oplus\left[V^{\otimes a_{r}} \otimes\left(V^{\vee}\right)^{\otimes b_{r}}\right],
$$

where $V^{\vee}$ is the dual to $V$. If $\omega: \mathcal{T} \longrightarrow k$-mod is a fiber functor, we define the monodromy group of $V$ at $\omega$ to be the Tannakian group scheme associated to the monodromy category via $\omega$. (See [3, Theorem 2.11]). 
Remark: It is not hard to show that if $\mathcal{T}=\operatorname{Rep}(G)$, where $G$ is an affine group scheme over $k$, then the monodromy category of $V \in \operatorname{Rep}(G)$ is equivalent to the category of representations of $\operatorname{Im}(G \longrightarrow \mathrm{GL}(V))$.

Definition 8. Let $\mathcal{T}$ be a Tannakian category over $k$. We say that $\mathcal{T}$ is finite if there exists an object $\Phi$ such that every object $M \in \mathcal{T}$ is a subquotient of some direct sum $\Phi^{\oplus a}$. (The terminology is justified by [3, Proposition 2.20].)

The following theorem, which will be proved in Section 4, implies Theorem 1 .

Theorem 9. For each $E \in \mathcal{T}(X)$, the Tannakian category $\langle E ; \mathcal{T}(X)\rangle_{\otimes}$ is finite (see Definition 8).

Proof that Theorem 9 implies Theorem 1: Take any $E \in \mathcal{T}(X)$, and let $G$ be the finite group scheme associated, via the fiber functor $x_{0}^{*}$, to the category $\langle E ; \mathcal{T}(X)\rangle_{\otimes}$. Hence, by the results in [9, $\S 2]$, there exists a $G$-torsor $P \longrightarrow X$ such that the functor

$$
P \times^{G}(\bullet): \operatorname{Rep}(G) \longrightarrow\langle E ; \mathcal{T}(X)\rangle_{\otimes},
$$

that send any $G$-module $V$ to the associated vector bundle $P \times^{G} V$, is an equivalence of Tannakian categories. But if $V$ is a finite dimensional representation of $G$, then [9, Proposition 3.8] shows that $P \times{ }^{G} V$ is essentially finite.

\section{The S-Fundamental group scheme and Reduction to the CASE of Curves}

We will show how to reduce the proof of Theorem 9 to the case where $X$ is of dimension one. This is possible due to a "Lefschetz Theorem" proved in [7].

\subsection{The $\mathbf{S}-$ fundamental group scheme [2], [7].}

Definition 10. The category $\operatorname{Vect}_{0}^{s}(X)$ is the full sub-category of $\mathbf{V B}(X)$ whose objects are strongly semistable vector bundles $V$ with

$$
\left[c_{1}(L)^{d-1} \cap c_{1}(V)\right]=\left[c_{1}(L)^{d-2} \cap \operatorname{ch}_{2}(V)\right]=0,
$$

where $L$ is a fixed polarization on $X$ and $d=\operatorname{dim} X$.

The category $\operatorname{Vect}_{0}^{s}(X)$ in Definition [10] is Tannakian [7, Proposition 5.4] and the fiber functor constructed using a $k$-point $x_{0}$ defines a fundamental group scheme:

$$
\pi\left(\operatorname{Vect}_{0}^{s}(X), x_{0}\right) \text {. }
$$

Let $\operatorname{Fr}_{X}: X \longrightarrow X$ be the absolute Frobenius morphism if $\operatorname{char}(k)>0$ and the identity map otherwise. 
If $E \in \mathcal{T}(X)$, then clearly $\operatorname{Fr}_{X}^{*} E$ is also in $\mathcal{T}(X)$. Hence, if $E$ is a vector bundle of $\mathcal{T}(X)$, then $\left(\operatorname{Fr}_{X}^{m}\right)^{*} E$ is Nori-semistable for every $m \in \mathbb{N}$; this entails that $E$ is strongly semistable with respect to the polarization $L$ on $X$. Using the projection formula for Chern classes, it is also clear that $c_{i}(E)$ is numerically trivial for any $E \in \mathcal{T}(X)$ and any $i>0$. Thus we have a natural fully faithful exact functor of Tannakian categories

$$
\mathcal{T}(X) \longrightarrow \operatorname{Vect}_{0}^{s}(X)
$$

By Corollary [5 (iii) and [3, Proposition 2.21], we have:

Lemma 11. The homomorphism of group schemes corresponding to (1)

$$
\pi\left(\operatorname{Vect}_{0}^{s}(X), x_{0}\right) \longrightarrow \pi\left(\mathcal{T}(X), x_{0}\right)
$$

is faithfully flat.

Proposition 12. Assume that Theorem 9 holds for curves. Then it holds for higher dimensional $X$.

Proof. We will proceed by induction. We assume that $\operatorname{dim}(X) \geq 2$ and that the theorem has been proved for all smooth projective varieties $C$ with $\operatorname{dim} C<\operatorname{dim} X$. We will now establish the existence of a smooth irreducible effective divisor $C \hookrightarrow X$ such that the natural homomorphism of group schemes

$$
\pi\left(\operatorname{Vect}_{0}^{s}(C), x_{0}\right) \longrightarrow \pi\left(\operatorname{Vect}_{0}^{s}(X), x_{0}\right)
$$

is faithfully flat. Due to [7, Theorem 10.2], it is enough to find a smooth, connected, ample effective divisor $C$ of high degree. We now apply Bertini's Theorem (see [4, p. 179, Theorem 8.18] and [4, 7.9.1, p. 245]) to $X$ embedded in $\mathbb{P}^{N}$ using the line bundle $L^{\otimes r}$.

Let $f: Y \longrightarrow X$ be a finite morphism from a variety $Y$ to $X$ and consider $E \in \mathcal{T}_{Y}(X)$. Clearly the restriction $\left.E\right|_{C}$ is an object of $\mathcal{T}(C)$. From Lemma 11 we know that the natural functors

$$
\langle E ; \mathcal{T}(X)\rangle_{\otimes} \longrightarrow\left\langle E ; \operatorname{Vect}_{0}^{s}(X)\right\rangle_{\otimes}
$$

and

$$
\left\langle\left. E\right|_{C} ; \mathcal{T}(C)\right\rangle_{\otimes} \longrightarrow\left\langle\left. E\right|_{C} ; \operatorname{Vect}_{0}^{s}(C)\right\rangle_{\otimes}
$$

are equivalences: they are fully faithful, their essential image is stable by subquotients and, by definition (see Definition 7), any object of the target category is a subquotient of an object from the source category. As the homomorphism in eq. (3) is faithfully flat, the functor

$$
\left\langle E ; \operatorname{Vect}_{0}^{s}(X)\right\rangle_{\otimes} \longrightarrow\left\langle\left. E\right|_{C} ; \operatorname{Vect}_{0}^{s}(C)\right\rangle_{\otimes}
$$

is also an equivalence (follows by repeating the previous argument). In conclusion, the Tannakian categories

$$
\langle E ; \mathcal{T}(X)\rangle_{\otimes} \quad \text { and } \quad\left\langle\left. E\right|_{C} ; \mathcal{T}(C)\right\rangle_{\otimes}
$$


are equivalent, and hence $\langle E ; \mathcal{T}(X)\rangle_{\otimes}$ is finite by the induction hypothesis.

\section{The CASE OF CuRve (CONClusion of PROOF)}

From now on we assume that $X$ is a smooth projective (connected) curve over $k$. This has the consequence that torsion free sheaves and vector bundles coincide.

\subsection{The maximal slope of certain coherent $\mathcal{O}_{X}$-algebras. Let}

$$
f: Y \longrightarrow X
$$

be a finite dominant morphism from a projective curve $Y$ and take any $E \in \mathcal{T}_{Y}(X)$, i.e., the vector bundle $f^{*} E$ is trivial. We assume that the extension of function fields provided by $f$ is separable (in other words, $f$ is generically étale). Let $\mathcal{A}$ denote the coherent $\mathcal{O}_{X}$-algebra $f_{*}\left(\mathcal{O}_{X}\right)$.

The following very simple observation is the key for all further considerations: Using the projection formula we have an isomorphism

$$
E \otimes_{\mathcal{O}_{X}} \mathcal{A} \cong \mathcal{A}^{\oplus r}
$$

This isomorphism induces a monomorphism of $\mathcal{O}_{X}$-modules

$$
\alpha: E \hookrightarrow \mathcal{A}^{\oplus r}
$$

Proposition 13. Let $\mathcal{A}_{\max }$ denote the maximal destabilizing subbundle of $\mathcal{A}$. Then the image of $\alpha$ is contained in $\left(\mathcal{A}_{\max }\right)^{\oplus r}$.

Proof. Evidently, the proposition will be proved once we establish that

$$
\mu_{\max }\left(\mathcal{A}^{\oplus r}\right)=\mu_{\max }(\mathcal{A})=0 .
$$

Note that it is enough to show that $\mu_{\max }(\mathcal{A}) \leq 0$.

Assume that $\mu_{\max }>0$, so that $\mathcal{A}_{\max }$ is semistable of positive slope. By adjointness, we have that

$$
\operatorname{Hom}_{Y}\left(f^{*}\left(\mathcal{A}_{\text {max }}\right), \mathcal{O}_{Y}\right)=\operatorname{Hom}_{X}\left(\mathcal{A}_{\text {max }}, \mathcal{A}\right) \neq 0 .
$$

But, by the separability hypothesis made on $f$, we know that $f^{*}\left(\mathcal{A}_{\max }\right)$ is $\left(f^{*} L\right)$-semistable of positive degree, so there are no non-zero homomorphisms from $f^{*}\left(\mathcal{A}_{\max }\right)$ to $\mathcal{O}_{Y}$. Hence $\mu_{\max }(\mathcal{A}) \leq 0$.

Proposition 13 has the following corollary: 
Corollary 14. Let $f: Y \longrightarrow X$ be as above. Then there exists a semistable locally free coherent $\mathcal{O}_{X}$-module $M:=\mathcal{A}_{\max }$ of degree zero such that for each $E \in \mathcal{T}_{Y}(X)$, there exists a monomorphism of $\mathcal{O}_{X}-$ modules

$$
\alpha_{E}: E \hookrightarrow M^{\oplus \operatorname{rank} E} .
$$

4.2. The case of separable (generically étale) morphisms. We continue with the above notation:

$$
f: Y \longrightarrow X
$$

is a finite surjective morphism from a projective curve $Y$ which induces a separable extension of function fields; $E$ is a vector bundle on $X$ such that $f^{*} E$ is trivial.

Recall that in Corollary 14, we showed that each $V \in\langle E ; \mathcal{T}(X)\rangle_{\otimes}$ is a sub-quotient of a direct sum of copies of a fixed torsion free semistable coherent sheaf of slope zero.

Theorem 15. Let $f: Y \longrightarrow X$ and $E$ be as above. Then the category $\langle E ; \mathcal{T}(X)\rangle_{\otimes}$ is finite. In particular, Theorem 9 (and hence Theorem 1) is true if $k$ has characteristic zero (see also the remark below).

Proof. As the subcategory $\mathcal{T}_{Y}(X)$ of $\mathcal{T}(X)$ is stable under sub-quotients (Proposition 4), we have $\left\langle E ; \mathcal{T}_{Y}(X)\right\rangle_{\otimes}=\langle E ; \mathcal{T}(X)\rangle_{\otimes}$. Let $M$ be the $\mathcal{O}_{X}$-module appearing in Corollary 14, so $M=\mathcal{A}_{\max }$. We want to find a vector bundle $\sigma(M) \in\left\langle E ; \mathcal{T}_{Y}(X)\right\rangle_{\otimes}$ which is a submodule of $M$ and which induces, for every $V \in\left\langle E ; \mathcal{T}_{Y}(X)\right\rangle_{\otimes}$, a factorization

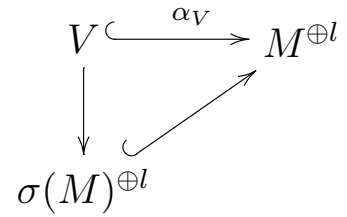

of the monomorphism $\alpha_{V}$ displayed in Corollary 14. By definition, this will prove that $\left\langle E ; \mathcal{T}_{Y}(X)\right\rangle_{\otimes}$ is finite.

Now let $N$ be an arbitrary semistable torsionfree sheaf on $X$ of slope zero. Let $\sigma(N) \subseteq$ $N$ be the largest sub-object of $N$ belonging to $\left\langle E ; \mathcal{T}_{Y}(X)\right\rangle_{\otimes}$; the existence of $\sigma(N)$ is guaranteed by the following two facts

(1) each ascending chain of sub-sheaves $N_{1} \subseteq N_{2} \subseteq \cdots \subseteq N$ must terminate;

(2) if $N_{1}$ and $N_{2}$ are sub-objects of $N$ belonging to $\left\langle E ; \mathcal{T}_{Y}(X)\right\rangle_{\otimes}$, then

$$
N_{1}+N_{2}=\operatorname{Im}\left(N_{1} \oplus N_{2} \longrightarrow N\right)
$$

must also be in $\left\langle E ; \mathcal{T}_{Y}(X)\right\rangle_{\otimes}$, due to Proposition 4. 
Let $V \in\left\langle E ; \mathcal{T}_{Y}(X)\right\rangle_{\otimes}$, and let $\alpha_{V}: V \hookrightarrow M^{\oplus l}$ be a monomorphism. It follows that $\alpha_{V}$ factors through the inclusion $\sigma\left(M^{\oplus l}\right) \subseteq M^{\oplus l}$. But again, using Proposition 4, we see that $\sigma\left(M^{\oplus l}\right)=\sigma(M)^{\oplus l}$.

Remarks: (1) In the proof of Theorem 15, we considered the largest sub-object lying in $\mathcal{T}_{Y}(X)$ of a torsionfree semistable sheaf of slope zero. This can be put in a more abstract setting: finding a right adjoint for the inclusion of $\mathcal{T}_{Y}(X)$ into the category of torsionfree semistables of slope zero. The important point is, of course, stability under quotients (Proposition 4). The reasoning is reminiscent of the construction of a right adjoint for the inclusion of categories $\operatorname{Rep}(H) \longrightarrow \operatorname{Rep}(G)$, where $G \longrightarrow H$ is surjective.

(2) In characteristic zero, there is also an easy proof of Theorem 1 which only assumes that the existence of a trace morphism

$$
\operatorname{Tr}_{\mathcal{A} / \mathcal{O}_{X}}: \mathcal{A} \longrightarrow \mathcal{O}_{X}
$$

(So normality of $X$ is already sufficient.) Such an $\mathcal{O}_{X}$-linear morphism allows us to find a section of the inclusion of $\mathcal{O}_{X}$ modules $\mathcal{O}_{X} \hookrightarrow \mathcal{A}$, so that, for $E \in \mathcal{T}_{Y}(X)$, each $E^{\otimes n}$ is a direct summand of $\mathcal{A}^{\oplus l}$. Hence, the indecomposable coherent $\mathcal{O}_{X}$-modules appearing in $E^{\otimes n}$ are isomorphic to certain indecomposable components of $\mathcal{A}$ (this uses the uniqueness of the Remak decomposition, see [1, p. 313, Theorem 1] and [1, p. 315, Theorem 2]); we then apply [9, Lemma 3.1] to conclude that $E$ is finite.

4.3. Proof of Theorem 9 in the case of curves. Let $E \in \mathcal{T}_{Y}(X)$, where $f: Y \longrightarrow X$ is a finite surjective morphism. We can assume without loss of generality that $Y$ is smooth and irreducible. Using the fact that the only purely inseparable morphisms between smooth curves are the Frobenia, we can find a factorization of $f$ as

$$
f=\operatorname{Fr}_{X}^{m} \circ g
$$

where $g: Y \longrightarrow X$ induces a separable extension of function fields. Then

$$
\left\langle\left(\operatorname{Fr}_{X}^{m}\right)^{*} E ; \mathcal{T}(X)\right\rangle_{\otimes}
$$

is finite due to Theorem 15. Let $G$ be the monodromy group of $E$ in $\mathcal{T}(X)$ (see Definition 7), and let

$$
\rho: G \longrightarrow \mathrm{GL}\left(x_{0}^{*} E\right)=\mathrm{GL}_{r}
$$

be the faithful monodromy representation. Denote by

$$
\varphi: k \longrightarrow k
$$

the arithmetic Frobenius $a \longmapsto a^{p^{m}}$ and by $\rho^{(m)}$ the twist of $\rho$ by $\varphi$; in concrete terms: if $\rho$ has matrix coefficients given by $\left(\rho_{i j}\right) \in \mathrm{GL}_{r}(\mathcal{O}(G))$, then the matrix coefficients of $\rho^{(m)}$ are $\left(\rho_{i j}^{p^{m}}\right)$. Let $G^{(m)}$ be the $k$-group scheme $G \otimes_{k, \varphi} k$; this is the scheme $G$ endowed with 
a different morphism to $\operatorname{Spec}(k)$. We have a commutative diagram of homomorphisms of $k$-group schemes

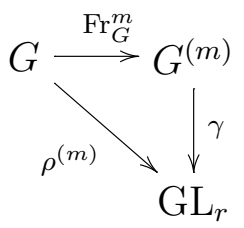

where $\gamma$ is defined by the matrix coefficients $\left(\rho_{i j}\right)$, now regarded as elements in $\mathcal{O}\left(G^{(m)}\right)$. It follows that $\operatorname{Ker}(\gamma)=\{1\}$, while $\operatorname{Ker}\left(\operatorname{Fr}_{G}^{m}\right)$ is a finite local group scheme (its ring of functions is a local Artin algebra). Since the representation

$$
\pi\left(\mathcal{T}(X), x_{0}\right) \longrightarrow G \stackrel{\rho^{(m)}}{\longrightarrow} \mathrm{GL}_{r}
$$

corresponds to $\left(\mathrm{Fr}_{X}^{m}\right)^{*} E$, the image of $\rho^{(m)}$ in $\mathrm{GL}_{r}$ is finite (recall that the first arrow above is faithfully flat). Hence, $G$ is an extension of finite group schemes, which shows that $G$ is a finite group scheme. We have proved Theorem 9 for curves and hence (see Proposition 12) for any smooth projective variety.

\section{REFERENCES}

[1] M. F. Atiyah, On the Krull-Schmidt theorem with application to sheaves, Bull. Soc. Math. Fr. 84 (1956), 307-317.

[2] I. Biswas, A. J. Parameswaran, and S. Subramanian, Monodromy group for a strongly semistable principal bundle over a curve, Duke Math. Jour. 132 (2006), 1-48.

[3] P. Deligne and J. Milne, Tannakian Categories, in: Hodge cycles, motives, and Shimura varieties (by P. Deligne, J. S. Milne, A. Ogus and K.-Y. Shih), pp. 101-228, Lecture Notes in Mathematics, 900, Springer-Verlag, Berlin-Heidelberg-New York, 1982.

[4] R. Hartshorne, Algebraic Geometry, Graduate Texts in Mathematics, No. 52, Springer-Verlag, New York, 1977.

[5] H. Lange and U. Stuhler, Vektorbündel auf Kurven und Darstellungen der algebraischen Fundamentalgruppe, Math. Zeit. 156 (1977), 73-83.

[6] A. Langer, Semistable sheaves in positive characteristic, Ann. of Math. 159 (2004), $251-276$.

[7] A. Langer, On the S-fundamental group scheme. Preprint May 2009.

[8] N. Nitsure, Construction of Hilbert and Quot schemes, in Fundamental algebraic geometry. Mathematical Surveys and Monographs, 123. American Mathematical Society, Providence, RI, 2005.

[9] M. V. Nori, On the representations of the fundamental group. Compositio Math. 33 (1976), 29-41. 
School of Mathematics, Tata Institute of Fundamental Research, Homi Bhabha Road, BOMBAY 400005, INDIA

E-mail address: indranil@math.tifr.res.in

Université de Paris 6, Institut de Mathématiques de Jussieu, 175, Rue du Chevaleret, 75013 Paris, France

E-mail address: dos-santos@math.jussieu.fr 